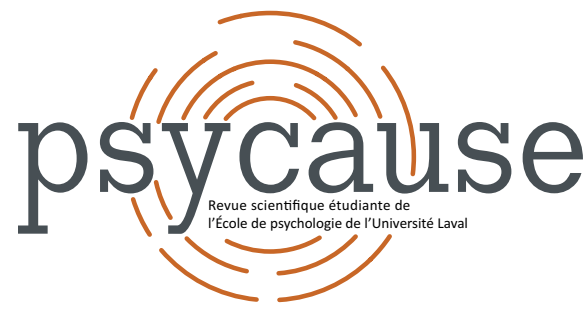

\title{
PSYCAUSE
}

Revue scientifique étudiante de l'École de psychologie de l'Université Laval

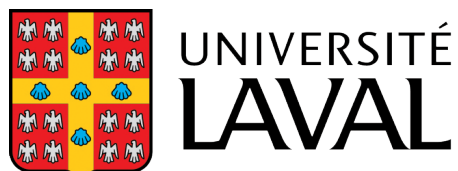

Faculté des sciences sociales École de psychologie

\section{ACCEPTABILITÉ DES TRAITEMENTS DE L'INSOMNIE UTILISANT LA PRIVATION DE SOMMEIL}

Charles ROCHETTE ${ }^{1}$, Léandre LAVOIE-HUDON', Erik CELIKOVIC ${ }^{1 *}$, Jacques LE BOUTHILLIER ${ }^{1}$ et Charles M. MORIN

1 École de psychologie, Université Laval

*erik.celikovic.1@ulaval.ca

\section{Pour citer l'article}

Rochette, C., Lavoie-Hudon, L., Celikovic, E., Le Bouthillier, J., \& Morin C.M. (2021). Acceptabilité des traitements de l'insomnie utilisant la privation de sommeil. Psycause: Revue scientifique étudiante de l'École de psychologie de l'Université Laval, 11(2), 3-4. 


\title{
ACCEPTABILITÉ DES TRAITEMENTS DE L'INSOMNIE UTILISANT LA PRIVATION DE SOMMEIL
}

\author{
Charles ROCHETTE ${ }^{1}$, Léandre LAVOIE-HUDON ${ }^{1}$, Erik CELIKOVIC ${ }^{1 *}$, Jacques LE BOUTHILLIER ${ }^{1}$ et Charles M. MORIN¹ \\ ${ }^{1}$ École de psychologie, Université Laval \\ *erik.celikovic.1@ulaval.ca
}

\section{Mots-clés : insomnie, traitement, réentraînement intensif au sommeil, acceptabilité, privation de sommeil totale}

La régulation du sommeil s'effectue par deux principaux processus biologiques. D'abord, les rythmes circadiens régulent le cycle veille-sommeil par le biais de processus hormonaux variant sur une période de 24 heures. Ensuite, la pression homéostatique entraîne l'augmentation du besoin de dormir en fonction de la durée de l'éveil (Borbély et al., 2016).

L'insomnie est un trouble du sommeil ayant une prévalence mondiale entre 10 et $15 \%$ et jusqu'à $40 \%$ de la population présente au moins un symptôme au moins trois fois par semaine (Morin et al., 2011). Le premier traitement recommandé pour l'insomnie est la thérapie cognitivocomportementale pour l'insomnie (TCCi; Cheung et al., 2019). Elle n'a toutefois pas d'effet significatif sur les symptômes chez 30 à $40 \%$ des patients (Morin et al. 2009). Cela pourrait s'expliquer en partie par un manque d'adhésion aux instructions causé par le délai de quelques semaines avant d'observer des résultats. En raison de ce délai, les patients peuvent devenir fatigués, de mauvaise humeur ou irritables (Kyle et al., 2011).

Un nouveau traitement, le réentraînement intensif au sommeil (RIS), vise des résultats rapides en misant sur une période d'intervention plus courte (24 heures) au cours de laquelle les patients tentent de s'endormir chaque 30 minutes.

Une privation de sommeil précédant l'intervention assure la hausse de la pression homéostatique, ce qui engendre des endormissements rapides favorisant l'association avec la chambre à coucher. Harris et ses collègues (2012) ont observé des résultats prometteurs jusqu'à six mois après le traitement. Toutefois, la durée de la privation d'environ $\mathbf{4 0}$ heures incluant l'intervention pourrait représenter un obstacle potentiel à l'adhésion en raison des effets transitoires, notamment de la somnolence et des émotions ressenties. Effectivement, une telle privation pourrait accentuer la réactivité émotionnelle (Simon et al., 2020). Le RIS compte également plusieurs facteurs (p. ex., nombreux réveils) influençant l'irritabilité (Toohey, 2020). L'objectif de cette étude est donc d'évaluer les niveaux de somnolence et d'émotions ressenties, ainsi que leur évolution lors d'un traitement RIS en comparaison à une privation de sommeil totale (PST) et à une nuit de sommeil.

\section{Méthode}

Dix participants souffrant d'insomnie chronique ont été assignés aléatoirement dans l'une des trois conditions: le RIS, la PST et la condition contrôle. Tous les participants passaient une première nuit de restriction de sommeil au laboratoire (23h00 à 5h30), puis la période d'intervention de 21 heures au laboratoire commençait la nuit suivante à 23h00. Pendant cette période, les participants de la condition RIS tentaient de s'endormir chaque 30 minutes, puis étaient réveillés après deux minutes. Les participants de la condition PST étaient totalement privés de sommeil. Les participants de la condition contrôle dormaient pendant les huit premières heures de la période d'intervention.

Les participants ont évalué leur somnolence et différentes émotions chaque heure pendant la période de 21 heures. L'échelle visuelle analogue utilisée contenait cinq souséchelles: somnolence, irritabilité, colère, tristesse et joie. Les participants devaient indiquer leur état sur un continuum de zéro (faible) à 10 (élevé). Les scores d'émotions étant corrélés, ils ont été regroupés en un seul score d'émotions négatives (joie inversée).

\section{Résultats et discussion}

Afin de tenir compte de certaines données manquantes (condition contrôle), une ANOVA à modèle linéaire mixte a été employée. Pour la somnolence, les résultats n'indiquent aucune différence significative entre la condition RIS et la PST, mais elles entraînent significativement plus de somnolence que la condition contrôle. Les courtes siestes de la condition RIS ne semblent pas réduire la somnolence des participants par rapport à une PST.

Pour les émotions, aucune différence significative n'a été observée entre les trois conditions. Ce résultat est surprenant compte tenu de la hausse d'irritabilité et de la réactivité émotionnelle qui était attendue avec le RIS et la PST. Ces résultats sont encourageants pour l'acceptabilité du RIS, puisque le traitement n'entraînerait pas plus d'émotions négatives qu'une simple PST ou qu'une nuit de sommeil.

Un autre aspect à considérer est la fluctuation des scores au cours de l'intervention. En effet, il serait possible que 
certains moments de l'intervention soient facilités par les rythmes circadiens, préparant l'individu à l'éveil (Borbély et al., 2016). Une analyse visuelle de la Figure 1 semble indiquer une baisse du niveau de somnolence entre environ 6 h00 et 9h00 le matin pour la condition RIS. La même conclusion ne peut cependant pas être établie pour les émotions (Figure 2).

\section{Figure 1.}

Niveau de somnolence selon l'heure et la condition pendant la période en laboratoire

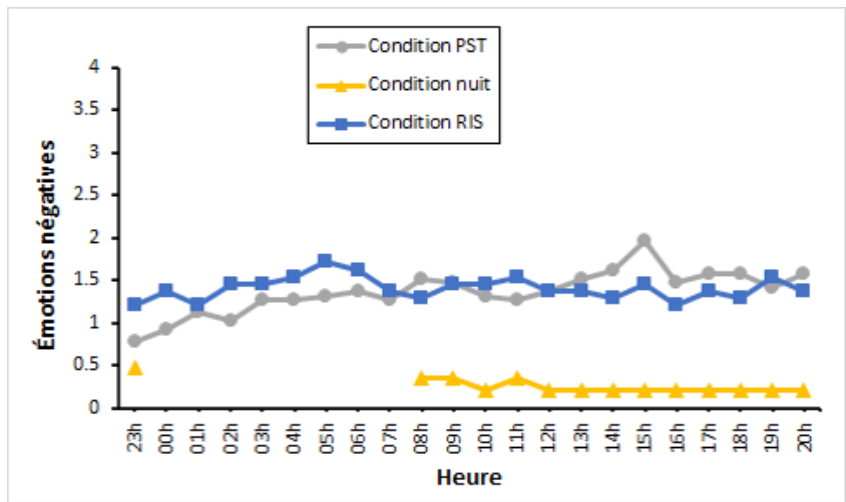

Figure 2.

Niveau d'émotions négatives selon l'heure et la condition pendant la période en laboratoire.

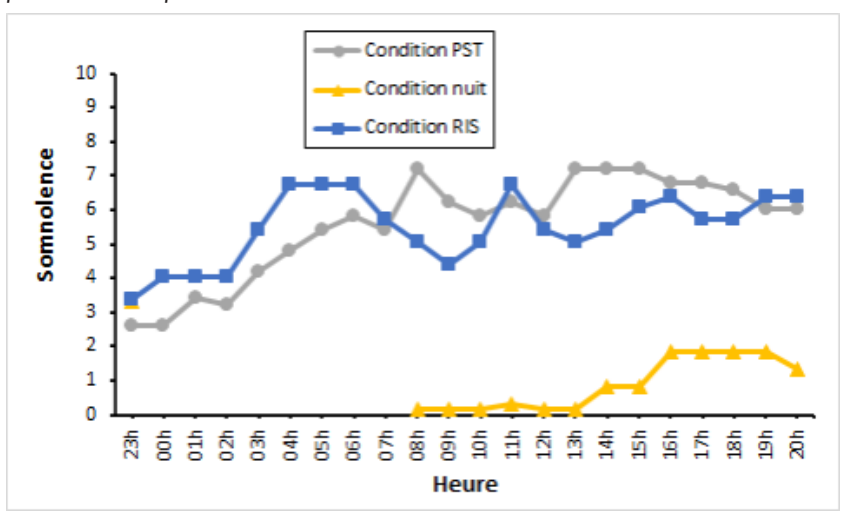

Bien que ces résultats soient encourageants pour l'acceptabilité du RIS, les études futures pourraient inclure d'autres mesures d'acceptabilité (p. ex., physiologiques) ou encore demander directement aux participants si le traitement est acceptable. II serait pertinent que d'autres protocoles plus réalistes (hors laboratoire) fassent l'objet d'une évaluation semblable.

\section{Références}

Borbély, A. A., Daan, S., Wirz-Justice, A., \& Deboer, T. (2016). The two-process model of sleep regulation: A reappraisal. Journal of Sleep Research, 25(2), 131-143. https://doi. org/10.1111/jsr.12371

Cheung, J. M. Y., Ji, X.-W., \& Morin, C. M. (2019). Cognitive behavioral therapies for insomnia and hypnotic medications: Considerations and controversies. Sleep Medicine Clinics, 14(2), 253-265. https://doi.org/10.1016/j.jsmc.2019.01.006

Kyle, S. D., Morgan, K., Spiegelhalder, K., \& Espie, C. A. (2011). No pain, no gain: An exploratory within-subjects mixedmethods evaluation of the patient experience of sleep restriction therapy (SRT) for insomnia. Sleep Medicine, 12(8), 735-747. https://doi.org/10.1016/j.sleep.2011.03.016

Harris, J., Lack, L., Kemp, K., Wright, H., \& Bootzin, R. (2012). A randomized controlled trial of intensive sleep retraining (ISR): A brief conditioning treatment for chronic insomnia. Sleep, 35(1), 49-60. https://doi.org/10.5665/sleep.1584

Morin, C. M., Vallières, A., Guay, B., Ivers, H., Savard, J., Mérette, C., Bastien, C., \& Baillargeon, L. (2009). Cognitive-Behavior Therapy, singly and combined with medication, for persistent insomnia: Acute and maintenance therapeutic effects. JAMA: the Journal of the American Medical Association, 301(19), 2005-2015. https://doi.org/10.1001/ jama.2009.682

Morin, C. M., LeBlanc, M., Bélanger, L., Ivers, H., Mérette, C., \& Savard, J. (2011). Prevalence of insomnia and its treatment in Canada, prevalence of insomnia and its treatment in Canada. The Canadian Journal of Psychiatry, 56(9), 540548. https://doi.org/10.1177/070674371105600905

Simon, E. B., Vallat, R., Barnes, C. M., \&Walker, M. P. (2020). Sleep loss and the socio-emotional brain. Trends in Cognitive Sciences, 24(6), 435-450. https://doi.org/10.1016/j. tics.2020.02.003

Toohey, M. J. (2020). Irritability characteristics and parameters in an international sample. Journal of Affective Disorders, 263, 558-567. https://doi.org/10.1016/j.jad.2019.11.021

\section{Pour citer l'article}

Rochette, C., Lavoie-Hudon, L., Celikovic, E., Le Bouthillier, J., \& Morin C.M. (2021). Acceptabilité des traitements de I'insomnie utilisant la privation de sommeil. Psycause: Revue scientifique étudiante de l'École de psychologie de l'Université Laval, 11(2), 3-4. 